\title{
New Approach to the Breaking of Space Symmetry in the Masses Spectrum Problem in the Aspect of Spontaneous Breaking of Space Symmetry
}

\author{
A. G. Syromyatnikov \\ Department of Physics, St. Petersburg University, Universitetskaya nab., St. Petersburg, Russia
}

Email address:

alsyromyatnikov@mail.ru

To cite this article:

A. G. Syromyatnikov. New Approach to the Breaking of Space Symmetry in the Masses Spectrum Problem in the Aspect of Spontaneous Breaking of Space Symmetry. International Journal of High Energy Physics. Special Issue: Breaking of Space Symmetry in the Masses Spectrum Problem. Vol. 3, No.1-1, 2016, pp. 15-21. doi: 10.11648/j.ijhep.s.2016030101.13

Received: October30, 2015; Accepted: October31, 2015; Published: June 2, 2016

\begin{abstract}
It is examined some analogy between properties of hypernucleus and supernucleus with properties of elementary particles and resonances electroproduction by spin shock-waves in ions beams. The serial theory considered processes of hypernucleus and supernucleus electroproduction for medium nuclei is complicated given the collective effects. All these calculations can be done in the t-channel reactions of hypernucleus and supernucleus electroproduction. So when deep inelastic scattering of leptons on a deuteron main contribution in the t-channel give the triangular diagram of anomalous Landau poles below of the two nucleon production threshold, containing in addition to nucleons and the particles insertion per a nucleon lines. Their admixture in the scattering cross-section is known, is low and the contribution of these charts in the scattering amplitude does not exceed $1 \%$. The properties of the deuteron vertex demonstrate that hypernucleus electroproduction not only $\Delta^{+}$, but also all other particles of this set in two nucleon systems are small. A similar approach in the t-channel has been developed and for heavy particles and resonances. In all cases, in particular for a deuteron and helium-3, a deviation of a parameter " $\mathrm{a}$ " as the masses ratio from $\mathrm{a}_{\mathrm{x}}=0.412$ accompanied by the suppression of contribution of the triangular anomalous Landau charts and as a consequence to a regarding smaller hypernucleus and supernucleus electroproduction. In the field of high-energy was studied formation of Galactic spin shock-waves. In all cases above $20^{\circ}$ Galactic longitude intensity of diffuse gamma radiation quickly subsides, so that the theory of Eight spin model profile compared with the intensity of Galactic diffuse gamma radiation from large gamma-ray space telescope (GLAST) is an experimental distribution of diffuse Galactic Gamma-ray bursts very accurately, in all range of the Galactic longitude within the accuracy of measurements in the presence of extra-galactic sources, Sails, etc. All of this confirms the conservation low of the spin angular momentum, as a part of a full and the isotropy of space-time across the Galaxy.
\end{abstract}

Keywords: Dilaton or Scale Symmetry, Dilaton Quark-Lepton X-Structure, Particles Photoproduction in Hypernucleus, Spin Shock-Wave

\section{Introduction}

Elementary particles and resonance production in high energy processes is characterized by scaling, when there is dilaton currents conservation low. It leads to the fundamental definition of lepton number as a quantum dilaton number (see for example [1]) such as lepton generations are distinguished on masses only. In another side in the analogy it may be possible dilaton quarks currents conservation too.

In previous works we showed in possibility processes of elementary particles and resonances electroproduction on a threshold of spin shock-waves (SSW) forming (see [1]) in accelerated beams of ions of some stable chemical elements. In that case it may be possible start from the simple fact of proportionality between all elementary particles masses and 24 resonance masses (see [1]) in one side and nuclei masses in another side with some constant coefficient to all-known elementary particles and resonances mass (see list at [1]).

There are more four works [1-4] at this direction and on the subject of the Special Issue of which mention should be 
made of the work [4], updates unfortunately in the Special Issue.

In that unexpected repeating of the whole by a fragments spectrum in 3 cases for particles and in 11 cases for resonances fit took admissible doubling of this calculated dilatation coefficient by means an ion electric charge doubling (in possibility processes of their electroproduction on a threshold of spin shock-waves (see[1]) forming [1] in accelerated beams of ions of some stable chemical elements; their photoproduction in hypernucleus and supernucleus also with one or two particles see[1]). Considerations of allknown resonances from particles list [1] according to doubling more 55 percents. Fundamentally in this case that $\mathrm{W}^{+}$boson of electroweak interaction in recalculating on mass directly join to a border of chemical elements stability through Bi-209.

In the work [1] we studied comparison analogy between properties of hypernucleus and supernucleus with properties of the elementary particles and resonances electroproduction by spin shock-waves in ions beams. The work was constructed by the following way. In the first it will be given statistic dates [1] for the linear dependence between series of 50 elementary particles masses and 121 resonances masses spectrum and corresponding nuclei atomic weights. Then we showed the analogy between properties of hypernucleus and supernucleus with properties of electroproduction of elementary particles and resonances by spin shock-waves in ions beams.

In ([1], Table 5) (see Table 1) we showed the analogy between properties of hypernucleus and supernucleus with properties of elementary particles and resonances electroproduction by spin shock-waves in ions beams. There was a special question: can we conclude from these data that is either on the ratio

$$
\frac{m_{E P, \text { Res }}}{M_{\text {Nucleous }}}=0.412
$$

Table 1. The analogy between properties of the proportionality between all-known elementary particles masses and resonance masses (see list: NAKAMURA K. et al. [Particle Date Groups], J PG 37, 075021 (2010) and 2011) in one side and nuclei masses in another side with some constant coefficient and properties of hipernucleus and supernucleus.

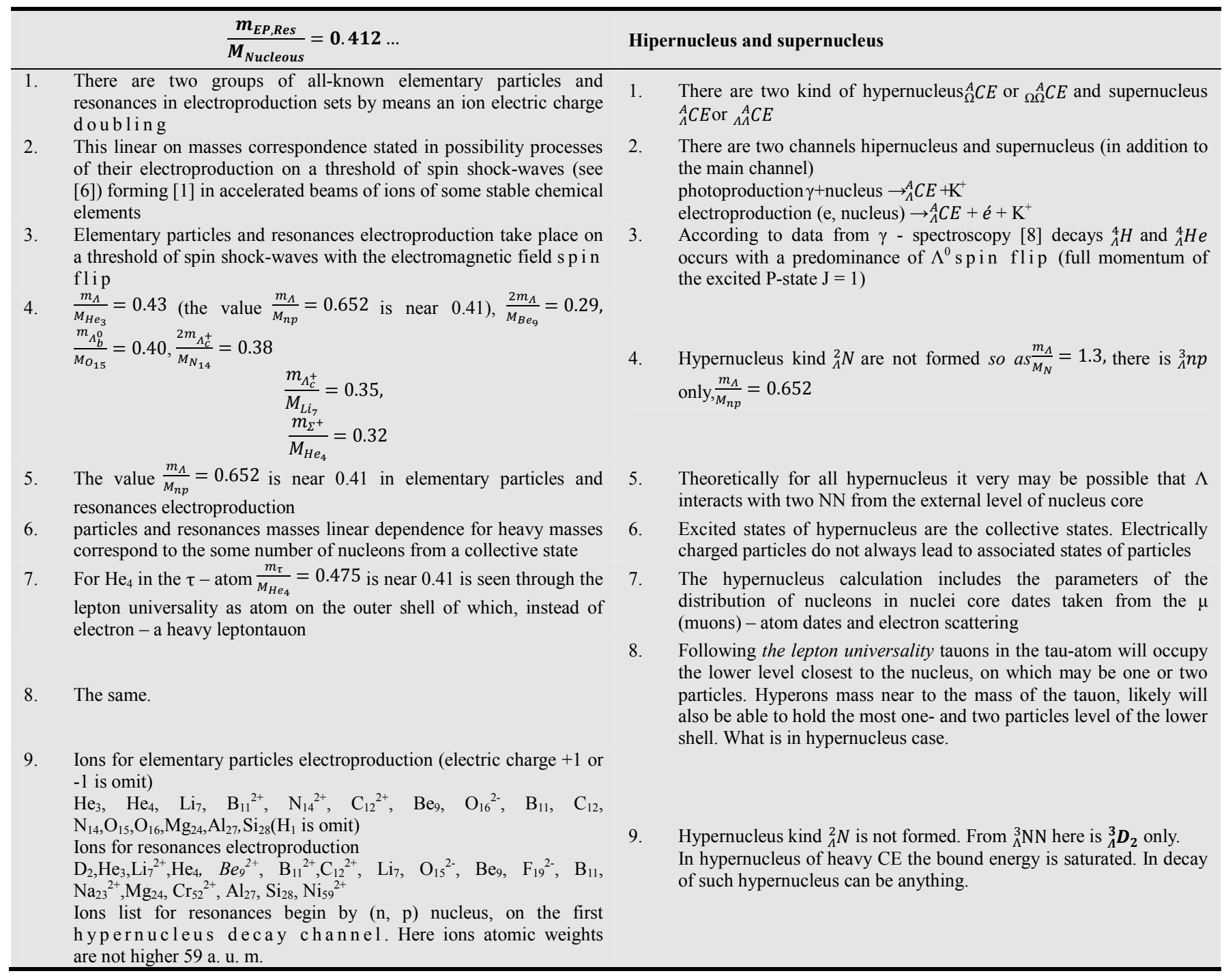


According to Table 1 it was concluded from the stated some analogy between properties of hypernucleus and supernucleus with properties of elementary particles and resonances electroproduction by spin shock-waves in ions beams that

1. Already lightest hypernucleus kind ${ }_{\Lambda}^{2} N$ are not formed so as $\frac{m_{\Lambda}}{M_{N}}=1.3$ (No 5), there is ${ }_{\Lambda}^{3} n p$ only. In this case $\frac{m_{\Lambda}}{M_{n p}}=0.652$ is near to the ratio $\frac{m_{E P, R e s}}{M_{\text {Nucleous }}}=$ $0.412 \ldots$ There are as a minimum eight supernucleus for which that ratio is fulfilled too. Theoretically for all hypernucleus it very may be possible that $\Lambda$ interacts with two NN from the external level of nucleus core. For $\mathrm{He}_{4}$ in the $\tau$-atom $\frac{m_{\tau}}{M_{\mathrm{He}_{4}}}=0.475$ is near 0.41 is seen through the lepton universality as atom on the outer shell of which, instead of electron - a heavy lepton tauon. The ions list for resonances ([1], Table 2) (see Table 2) begin by (n,p) nucleus, on the first hypernucleus decay channel - on $\mathrm{D}_{2}$.

2. There are two kind of hypernucleus ${ }_{\Omega}^{A} C E$ or ${ }_{\Omega \Omega}^{A} C E$ and supernucleus ${ }_{\Lambda}^{A} C E$ or ${ }_{\Lambda \Lambda}^{A} C E$ also as there are two groups of all-known elementary particles and resonances in electroproduction sets by means an ion electric charge doubling. Theoretically for all hypernucleus it very may be possible that $\Lambda$ interacts with two NN from the external level of nucleus core. Following the lepton universality tauons in the tau-atom will occupy the lower level closest to the nucleus, on which may be one or two particles. Hyperons mass near to the mass of the tauon, likely will also be able to hold the most one- and two particles level of the lower shell. What is in the hypernucleus case also.

According to data from $\gamma$-spectroscopy (see [1]) decays ${ }_{\Lambda}^{4} \mathrm{H}$ and ${ }_{\Lambda}^{4} \mathrm{He}$ occurs with a predominance of $\Lambda^{0}$ spin flip (full momentum of the excited P-state $J=$ $1)$. This is consistent with the fact that in the elementary

Table 2. All discovered 121 resonances group in some sets for every ion kind on that occur their electroproduction.

\begin{tabular}{|c|c|c|c|}
\hline $\mathbf{C E}^{1}$ & $\mathrm{~A}^{2}$ & $\mathbf{B}^{3}$ & Resonances \\
\hline $\begin{array}{l}\mathrm{D}_{2} \\
771\end{array}$ & 4 & $748 \pm 145$ & $\begin{array}{l}\rho^{+}[770] K^{*}[892] \omega[783] \\
\eta[548]\end{array}$ \\
\hline $\begin{array}{l}\mathrm{He}_{3} \\
1157\end{array}$ & 9 & $1102 \pm 115$ & $\begin{array}{l}\mathrm{f}_{0}[980] \mathrm{a}_{0}[980] \varphi[1019] \\
\eta_{1}[1170] \mathrm{a}_{1}[1230] \mathrm{b}_{1}[1230] \\
\mathrm{f}_{1}[1282] \mathrm{K}_{1}[1270] \mathrm{f}_{2}[1270] \\
\Delta_{0}[1230] \Sigma^{0}[1193] \Sigma^{+}[1190]\end{array}$ \\
\hline & 6 & $1241 \pm 62$ & $\begin{array}{l}\Sigma[1197] \Xi^{0}[1314] \Xi^{0}[1322] \\
\eta[1294] \pi[1300] \mathrm{a}_{2}[1320]\end{array}$ \\
\hline $\mathrm{Li}_{7}^{2+}$ & 4 & $1320 \pm 35$ & $f_{0}[1370]$ \\
\hline 1338 & 5 & & $\begin{array}{l}\mathrm{K}_{1}[1400] \pi[1400] \mathrm{K}_{0}^{*}[1430] \\
\mathrm{K}_{2}^{*}[1430] \mathrm{f}_{1}[1426]\end{array}$ \\
\hline $\begin{array}{l}\mathrm{He}_{4} \\
1542\end{array}$ & 6 & $1533 \pm 75$ & $\begin{array}{c}\rho[1450] \eta[1476] \mathrm{f}_{0}[1500] \\
\mathrm{f}_{2}[1525] \pi_{1}[1600] \pi_{2}[1645] \\
\Sigma[1385] \Sigma[1530] \Lambda[1405]\end{array}$ \\
\hline & 4 & $1468 \pm 126$ & $\omega[1650]$ \\
\hline & 10 & $1710 \pm 50$ & $\begin{array}{l}\pi_{2}[1670] \omega_{3}[1670] \varphi[1680] \\
\mathrm{K}^{*}[1680] \rho_{3}[1700] \mathrm{f}_{0}[1710] \\
\mathrm{K}_{3}[1770] \pi[1800] \chi[1835] \\
\mathrm{K}_{2}[1820]\end{array}$ \\
\hline $\mathrm{Be}_{9}{ }^{2+}$ & 2 & & $\varphi_{3}[1850] \pi_{2}[1880]$ \\
\hline 1724 & 7 & $1734 \pm 65$ & $\begin{array}{l}\Sigma[1650] \Sigma[1670] \\
\Xi[1690] \\
\Sigma[1750] \Lambda[1800] \Lambda[1810] \\
\mathrm{K}_{2}[1770]\end{array}$ \\
\hline & 3 & $1840 \pm 40$ & $\begin{array}{l}\Lambda[1820] \Xi[1820] \Lambda[1890] \\
\Sigma[1915] \Sigma[1940]\end{array}$ \\
\hline $\begin{array}{l}\mathrm{B}_{11}^{2+} \\
1931\end{array}$ & 8 & $1968 \pm 45$ & $\begin{array}{l}\Xi[1950] \Sigma[2030] \\
\Xi[2030] \Lambda[2100] \\
\mathrm{f}_{2}[1950] \mathrm{f}_{2}[1959] \\
\Lambda[2110] \mathrm{a}_{4}[2040] \mathrm{D}^{*}[2007]\end{array}$ \\
\hline & 8 & $2039 \pm 37$ & $\begin{array}{l}D^{*}[2010] f_{2}[2010] f_{4}[2050] \\
K_{4}^{*}[2045] \varphi[2170]\end{array}$ \\
\hline & 3 & $2283 \pm 55$ & $\Sigma[2250] \Omega[2250] \Lambda[2350]$ \\
\hline $\begin{array}{l}\mathrm{C}_{12}{ }^{2+} \\
2145\end{array}$ & 6 & $2280 \pm 80$ & $\begin{array}{l}\mathrm{f}_{2}[2300] \mathrm{f}_{2}[2340] \\
\mathrm{D}^{*}{ }_{\mathrm{s} 0}[2317] \mathrm{D}_{2}[2160] \\
\mathrm{D}_{\mathrm{s1} 1}[2460] \mathrm{D}_{1}[2420]\end{array}$ \\
\hline $\begin{array}{l}\mathrm{Li}_{7} \\
2676\end{array}$ & 6 & $2582 \pm 43$ & $\begin{array}{l}\Sigma_{\mathrm{c}}[2520] \Lambda_{\mathrm{c}}[2595] \\
\Lambda_{\mathrm{c}}[2625] \Xi_{\mathrm{c}}[2645] \\
\mathrm{D}_{\mathrm{s} 1}[2536] \mathrm{D}_{\mathrm{s} 2}[2572]\end{array}$ \\
\hline $\mathrm{O}_{15}{ }^{2-}$ & 7 & $2916 \pm 70$ & $\Xi[3080] \Xi_{\mathrm{c}}[2800]$ \\
\hline
\end{tabular}




\begin{tabular}{|c|c|c|c|}
\hline$\overline{C E^{1}}$ & $\overline{A^{2}}$ & $\mathbf{B}^{3}$ & Resonances \\
\hline 2868 & & & $\begin{array}{l}\Lambda_{\mathrm{c}}[2880] \Lambda_{\mathrm{c}}[2940] \\
\Xi[2980] \\
\eta_{\mathrm{c}}(1 \mathrm{~S} 1)[2980] \mathrm{J} / \psi[3097]\end{array}$ \\
\hline $\begin{array}{l}\mathrm{Be}_{9} \\
3447\end{array}$ & 5 & $3502 \pm 60$ & $\begin{array}{l}\chi_{c 0}[3415] \chi_{c 1}(1 \mathrm{P})[3511] \\
\chi_{c 2}(1 \mathrm{P})[3556] \mathrm{h}_{\mathrm{c}}(1 \mathrm{P})[3525] \\
\eta_{\mathrm{c}}(2 \mathrm{~S})[3637]\end{array}$ \\
\hline $\begin{array}{l}\mathrm{F}_{19}^{2-} \\
3639\end{array}$ & 1 & & $\psi(2 s)[3626]$ \\
\hline $\begin{array}{l}\mathrm{B}_{11} \\
3862\end{array}$ & 3 & $3894 \pm 140$ & $\psi[3770] \chi[3872] \psi[4040]$ \\
\hline $\begin{array}{l}\mathrm{Na}_{23}{ }^{2+} \\
4348\end{array}$ & 3 & $4280 \pm 130$ & $\begin{array}{l}\psi^{\prime \prime \prime}[4160] \chi[3872] \\
\psi[4415]\end{array}$ \\
\hline $\begin{array}{l}\mathrm{Mg}_{24.3} \\
9298\end{array}$ & 1 & & $\gamma[9460]$ \\
\hline $\begin{array}{l}\mathrm{Cr}_{52}^{2+} \\
9925\end{array}$ & 3 & $9930 \pm 89$ & $\begin{array}{l}\chi_{b 0}(1 \mathrm{P})[9856] \chi_{\mathrm{b2}}(1 \mathrm{P})[9912] \\
\gamma(2 \mathrm{~S})[10023]\end{array}$ \\
\hline $\begin{array}{l}\mathrm{Al}_{27} \\
10306\end{array}$ & 4 & $10251 \pm 80$ & $\begin{array}{l}\gamma(1 \mathrm{D})[10163] \\
\chi_{b 0}(2 \mathrm{P})[10232,5] \\
\chi_{b 1}(2 S)[10255,4] \gamma(3 S)[10355]\end{array}$ \\
\hline $\begin{array}{l}\mathrm{Si}_{28} \\
10688\end{array}$ & 2 & $10720 \pm 140$ & $\gamma(4 \mathrm{~S})[10580] \gamma[10860]$ \\
\hline $\begin{array}{l}\mathrm{Ni}_{59}{ }^{2+} \\
11201\end{array}$ & 1 & & $\gamma[11020]$ \\
\hline average & & $\pm 70 \mathrm{MeV}$ & $\begin{array}{l}\text { Average distinguish between electroproduction threshold and set centre } \\
28 \pm 85 \mathrm{MeV}\end{array}$ \\
\hline
\end{tabular}

${ }^{1}$ Number of particles in the set

${ }^{2}$ Standard deviation on distinguish between particles masses, $\mathrm{MeV}$

${ }^{3}$ Ions of chemical elements (electric charge +1 or -1 is omitted) Electroproduction threshold, $\mathrm{MeV}$

particles and resonances electro-production take place on a threshold of spin shock-waves with the electromagnetic field s pin-flip.

3. Stated in [1] the fact of repeating of all-known elementary particles and resonances mass spectrum as whole of nuclei atomic weight of ions of some stable chemical elements with a coefficient in proportion to $\mathrm{M} / \mathrm{e}$ for an ion is signed that all-known elementary particles and resonances masses also as masses of stable chemical elements and isotopes are defined by positions of features - jumps of a quark-lepton dilaton $\mathrm{X}$-structure (see [1]). Whereas the definition of a value of the ratio between the masses of particles and nuclei requires taking into account the contribution of SSWCnumber components of classical interact.

According to the quark-lepton $\mathrm{X}$-structure theory $[1,5,6]$ there are following components of nucleus - nucleons, $\alpha-$ particles and the 9 nucleons compound $\left(\mathrm{Be}_{9}\right)$ with one muon - from which you can compile any stable nucleus. In the hypernucleus hyperon (with the mass near the tauon mass) interacts with these components. In addition, the mass of any stable nucleus is equal to integer number of the tauon mass (with the Standard Deviation \pm 0.5 a.u.m.). Therefore according to [1], the relationship of the masses of all the particles also defined in integer numbers. Thus it was shown that the spectrum of the masses of all-known particles and resonances of the theoretically calculated through the quarklepton X-structure theory [5] for the masses of nuclei.

\section{New approach}

From the stated analogy between some properties of hypernucleus and supernucleus with properties of elementary particles and resonances electroproduction by spin shockwaves in beam ions it is clear that the serial theory considered processes of hypernucleus and supernucleus electroproduction for medium nuclei is complicated given the collective effects. On the other hand all these calculations can be done in the t-channel reactions of hypernucleus and supernucleus electroproduction. So when deep inelastic scattering of leptons on a deuteron main contribution in the tchannel give the triangular diagram Fig. 1 of anomalous Landau poles $t_{0}$ below of the two nucleon production threshold, containing in addition to nucleons and the particles insertion per a nucleon lines. This insert is valid, because change of the isospin in this case does not exceed 1. In additional, there is a new isospin shock - wave (see $[5,6])$ in deep inelastic rescattering processes of the cumulative mesons production in the deuteron where the gravitation mass defect [7] $\sim 10 \mathrm{MeV}$ in the proton formfactor is required for distancing of the scaling parameter far from the kinematically forbidden zone. Isospin (I) -flip effect occur with increasing momentum in the lepton scattering being in the deuteron structure on $90 \%$ on a proton $(\mathrm{I}=+1 / 2)$ changes by the neutron $(I=-1 / 2)$ on $90 \%$ also [8]. As you know a deep inelastic scattering of the iron nucleus on a deuteron in 


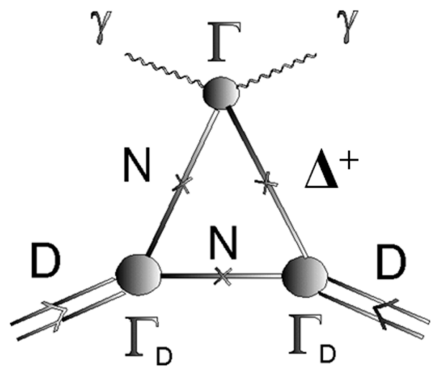

Fig. 1. The triangular anomalous Landau diagram of deep inelastic scattering of leptons (photon exchange) on a deuteron in the t-channel. $\Gamma$ is a Photon, $D$ is a deuteron, $N$ is a nucleon, $\Delta^{+}$is an elementary particles, $\Gamma$ is a nucleon vertex, $\Gamma_{D}$ is a deuteron vertex, "cross" means the particle on the surface of the masses.

a cumulative area comes also as on a single nucleon. This confirms the calculated conclusion [5] about the dominance of the deposits of individual nucleons in structural function of deuteron in cumulative area.

Their admixture in the scattering cross-section is known, is low and the contribution of these charts in the scattering amplitude does not exceed $1 \%$. The properties of the deuteron vertex demonstrate that hypernucleus electroproduction not only $\Delta^{+}$, but also all other particles of this set ([1], Table 1) (see Table 3) in two nucleon systems are small. A parameter "a" as the masses ratio

as you would expect.

$$
a=\frac{m_{E P, \text { Res }}}{M_{\text {Nucleous }}}=0.6 \sim \mathrm{a}_{\mathrm{X}}=0.412
$$

Table 3. All discovered 46 elementary particles group in some sets for every ion kind on that occur their electroproduction.

\begin{tabular}{|c|c|c|c|}
\hline $\mathbf{C E}^{1}$ & $\mathbf{A}^{2}$ & $\mathbf{B}^{3}$ & Particles \\
\hline $\mathrm{p}^{+}$ & 3 & \pm 32 & $\mathrm{~K}^{+}[493,7] \mathrm{K}_{\mathrm{LS}}^{0}[497,6] \eta^{0}[547,8]$ \\
\hline $\mathrm{He}_{3}$ & 8 & \pm 68 & $\begin{array}{l}\Delta^{0}, \Delta^{+}[1232] \Lambda^{0}[1232] \Sigma^{+}[1189,4] \\
\Sigma^{0}[1192,5] \Sigma^{-}[1197,4] \Xi^{0}[1315] \\
\Xi^{-}[1321]\end{array}$ \\
\hline $\mathrm{He}_{3}$ & 3 & \pm 170 & $\mathrm{p}^{+} \mathrm{n}[938] \Delta^{++}[1232]$ \\
\hline $\mathrm{He}_{3}$ & 1 & - & $\varphi[1019]$ \\
\hline $\mathrm{He}_{4}$ & 1 & - & $\Omega^{-}[1672]$ \\
\hline $\mathrm{Li}_{7}$ & 1 & - & $\Omega_{\mathrm{c}}^{0}[2698]$ \\
\hline $\mathrm{B}_{11}^{2+}$ & 1 & - & $\mathrm{D}[1870]$ \\
\hline $\mathrm{N}_{14}^{2+}$ & 6 & \pm 110 & $\begin{array}{l}\Lambda_{\mathrm{c}}^{+}[2284] \Lambda_{\mathrm{c}}^{0}[2274] \Xi_{\mathrm{c}}^{+}[2466] \\
\Xi_{\mathrm{c}}^{0}[2472] \Lambda_{\mathrm{c}}^{-}[2284] \Xi_{\mathrm{c}}^{-}[2466]\end{array}$ \\
\hline $\mathrm{C}_{12}^{2+}$ & 2 & \pm 51 & $\mathrm{D}^{*}[2010] \mathrm{D}_{\mathrm{s}}^{*}[2112]$ \\
\hline $\mathrm{Be}_{9}$ & 1 & - & $\eta_{\mathrm{c}}[3592]$ \\
\hline $\mathrm{O}_{16}{ }^{2-}$ & 2 & \pm 68 & $\mathrm{~J} / \psi[3097] \eta_{\mathrm{c}}[2980]$ \\
\hline $\mathrm{B}_{11}$ & 2 & \pm 42 & $\Psi^{\prime}[3685] \Psi^{\prime \prime}[3768]$ \\
\hline $\mathrm{C}_{12}$ & 1 & - & $\Psi^{\prime \prime \prime}[4415]$ \\
\hline $\mathrm{N}_{14}$ & 3 & \pm 45 & $\mathrm{~B}^{*}[5325] \mathrm{B}_{\mathrm{s}}^{0}[5366] \mathrm{B}_{\mathrm{s}}^{*}[5415]$ \\
\hline $\mathrm{O}_{15}$ & 7 & \pm 104 & $\begin{array}{l}\Lambda_{\mathrm{b}}^{0}[5624] \mathrm{B}_{\mathrm{s} 1}[5830] \mathrm{B}_{\mathrm{s} 2}[5840] \\
\Xi_{\mathrm{b}}[5629,6] \mathrm{B}_{1}[5721] \bar{\Lambda}_{h}^{0}[5624] \\
\Xi_{h}[5629,6]\end{array}$ \\
\hline $\mathrm{O}_{16}$ & 2 & 0 & $\mathrm{~B}_{\mathrm{c}}^{+}[6277] \mathrm{B}_{\mathrm{c}}^{-}[6277]$ \\
\hline $\mathrm{Mg}_{24}$ & 1 & - & $\mathrm{Y}[9459,7]$ \\
\hline $\mathrm{Al}_{27}$ & 2 & \pm 166 & $\mathrm{Y}^{\prime}[10018] \mathrm{Y}^{\prime \prime}[10350]$ \\
\hline $\mathrm{Si}_{28}$ & 1 & - & $\mathrm{Y}^{\prime \prime \prime}[10573]$ \\
\hline average & & $\pm 78 \mathrm{MeV}$ & \\
\hline
\end{tabular}

In the following case of three nucleon system of helium-3 deep inelastic scattering when chart of leptons on a helium-3 main contribution in the t-channel will also contain the triangular diagram of anomalous Landau poles type Fig. 1, where each deuteron line comes from the helium-3 vertex with the nucleon or $\Delta^{+}$insertions. In this case from one side the masse ratio $\mathrm{a}=0.44$ is very more nearly to $\mathrm{a}_{\mathrm{x}} 0.412$ than for the deuteron $\mathrm{a}=0.6$. In another side qualitatively for a helium-4 or 5 its vertex will give a significantly smaller contribution to the scattering cross-section because of the greater weight of the inserted component. Accordingly, this and hypernucleus electroproduction is significantly suppressed up to zero. And this is for the case of large values of $\mathrm{a}=0.58$ for a helium- 4 and $\mathrm{a}=0.73$ for helium-5. This applies to hypernucleus electroproduction not only $\Delta^{+}$, but also all other particles of this set of Table 3 .

A similar approach in the t-channel can be developed and for heavy particles and resonances. So when deep inelastic scattering of leptons on a nitrogen-14 main contribution in the $\mathrm{t}$-channel give the triangular diagram type Fig. 1 of anomalous Landau poles $t_{0}$ below of the two alpha-particles production threshold, containing in addition to nucleons and the $\mathrm{B}^{*}[5325]$ particles insertion per a 5-nucleon states lines instead of the nucleon and $\Delta^{+}$and beryllium-10 instead of a deuteron with an appropriate redefinition of vertices. This diagram should dominate for the masses due to the proximity of the masses $\mathrm{B}^{*}[5325]$ and a 5-nucleons state also as for a deuteron with its low binding energy. Largest mass parameter 0.405 compared with a cases of carbon-13 and oxygen-15 with inserts, respectively beryllium-9 and beryllium-11, for which "a" is equal to 0.437 and 0.38 , you can expect a significantly smaller contribution to the scattering crosssection, as it will be cause of the increasing pole enough diagrams Landau $t_{0}$ due to a large difference in the masses. This applies to supernucleus electroproduction not only $\mathrm{B}^{*}[5325]$, but also all other particles $\mathrm{B}_{\mathrm{s}}^{0}[5366]$ and $\mathrm{B}_{\mathrm{s}}{ }^{*}[5415]$ of this set of Table 3 .

A border of chemical elements stability through Bi-209 is defined by possible processes of electroproduction on a threshold of spin shock-waves forming in accelerated beams of ions radioactive $\mathrm{Po}$ and At on a mass of $\mathrm{W}^{+}$boson of electroweak interaction.

\section{When Anisotropy of Vacuum Set the Space Isotropy}

Many shared the view that, in determining the spectrum of masses should be taken of the gravity. All this investigation can be directly generalized for the case of the space strings in terms of the cone space topology which has no difference from the plane space-time everywhere except for a small angle, which is proportional to the mass density of the string by Nilsen-Olsen, which is similar to the mass angle of leptons anisotropy (see [8]) in the author`s Conformal Gauge Theory of Gravitation (1996). Standard Model, taking into account the gravity in evitably accompanied by anisotropy of 
vacuum [7] that whatever was said about the geometry of space-time. On the other hand the globality of spatial anisotropy is due to the conformal symmetry.

Results of some theoretical and experiment investigations of space-time anisotropy of Galactic gamma-rays angular distribution within the frame of the space rays generation mechanism on a method of direct transformation of intergalactic gamma-rays to the current on spin shock-waves are presented in [2, 3, 9]. However, the source anisotropy origin is unknown. Here it is shown that on Fermi telescope data brightness at the edge of the area, limited $20^{\circ}$ Galactic longitude, twice higher the brightness of diffuse eradiation. Thus the range of the observed distribution mapping of gamma rays from the Galactic longitude of theoretical (intensity of above-average level) in fact is limited above $20^{\circ}$ strict accordance with the theory. In [2] found that the Galactic gamma-ray angular anisotropy axis has following coordinates: longitude $1=96^{\circ}$, latitude $\approx 20^{\circ}$, that corresponds in the second equatorial coordinate system: right ascension $\alpha=271^{\circ}$, declination $\delta \approx 40$-supernova residuals Cygnus X-3 on periphery.

Additionally to [2] in [3] it was shown that after the mass of the muon and tau-neutrinos calculation we came to the same but already the integer 2:5 $=0.4$ ratio between the mass of muon neutrinos and muon ultra-light weight torsion [8]. Found that the calculated mass of the tau-neutrino is the same as the largest with heat $3 / 2 \mathrm{~T}$ relic neutrinos. In the standard model $\mathrm{T}=2 \mathrm{~K}$. Considering the temperature of the relic neutrinos as the effective gravitational temperature $\mathrm{T} \Gamma=$ $2 / 9 \mathrm{M}$ for some mass $\mathrm{M}$, it is fallowed the ratio $\mathrm{M}=$ $3 m_{v_{\tau}}$, corresponding to reactions of the dissolution of the relic neutrinos by three sterile active Tau-neutrinos.

Solar neutrino flux measurements data and its temporal variations in the Chlorine-Argon experiment for the period 1970-1994, examined with particular attention near dated frontiers of solar activity (FSA), which defined by means of the key criterion of $2 \ln 2$ in point a significant manifestation of the eccentric planets influence to the Sun. Review shall be carried out within the framework of the standard model, taking into account the gravity. The results are presented in the form of pie charts in a consecutive temporary scan on a direct ascent of Earth's orbital position $\alpha$, recalculated according to the date of the middle of each of the series of measurements. The corners of the sectors $35^{\circ}$ and more defined for temporary length of the each measurement. The amplitude of the sector is equal to the measured magnitude of neutrino flow minus the constant component 2.57 SNU. All charts show the priority direction of the solar neutrino flux detector checked by angles $\alpha=271^{\circ}, \delta=40^{\circ}\left(-15^{\circ}\right)$ axis of anisotropy of Galactic Gamma-radiation, as well as a number of less significant in transverse to the axis of the direction. Researched the relationship between solar activity, seismic activity of the Earth and neutrinos flows registered in chlorine-argon experiment for the period 1970-1994, as well as in the SAGE for the period 1970-2008 years. There are correlations of solar activity with seismic activity of the Earth and the positive correlation between seismic activity of the Earth and the neutrino flow variations, reaching 95\% when taking into account the peculiarities of the matching of dates of solar maximums with FSA dates 1978, 1993-5 and 2002. It turned out that all known and perhaps predicted on 2018 year the greatest average annual values of the flow of solar cosmic rays which defines corresponding dose fall exactly on the years of FSA defined on the planets eccentric.

\section{Conclusion}

1) Thus it was shown that the spectrum of the masses of all-known particles and resonances of the theoretically calculated through the quark-lepton X-structure theory [5] for the masses of nuclei.

2) We shown in possibility processes of elementary particles and resonances electroproduction on a threshold of spin shock-waves (SSW) forming in accelerated beams of ions of some stable chemical elements.

3) Stated in [1] the fact of repeating of all-known elementary particles and resonances mass spectrum as whole of nuclei atomic weight of ions of some stable chemical elements with a coefficient in proportion to $\mathrm{M} / \mathrm{e}$ for an ion is signed that all-known elementary particles and resonances masses also as masses of stable chemical elements and isotopes are defined by positions of features-jumps of a quark-lepton dilaton $\mathrm{X}$-structure (see [1]). Whereas the definition of a value of the ratio between the masses of particles and nuclei requires taking into account the contribution of SSWCnumber components of classical interact.

4) According to a quark-lepton $X$-structure theory $[1,5,6]$ there are following components of nucleus -nucleons, $\alpha$-particles and the 9 nucleons compound $\left(\mathrm{Be}_{9}\right)$ with one muon -from which you can compile any stable nucleus. In the hypernucleus hyperon (with the mass near the tauon mass) interacts with these components. In addition, the mass of any stable nucleus is equal to integer number of the tauon mass

5) The serial theory considered processes of hypernucleus and supernucleus electroproduction for medium nuclei is complicated given the collective effects. On the other hand all these calculations can be done in the t-channel reactions of hypernucleus and supernucleus electroproduction. So when deep inelastic scattering of leptons on a deuteron main contribution in the t-channel give the triangular diagram Fig. 1 of anomalous Landau poles $\mathrm{t}_{0}$ below of the two nucleon production threshold, containing in addition to nucleons and the particles insertion per a nucleon lines. Their admixture in the scattering cross-section is known, is low and the contribution of these charts in the scattering amplitude does not exceed 1\%. The properties of the deuteron vertex demonstrate that hypernucleus electroproduction not only $\Delta^{+}$, but also all other particles of this set of Table 3 in two nucleon systems are small. A parameter "a" as the masses ratio 


$$
a=\frac{m_{E P, \text { Res }}}{M_{\text {Nucleous }}}=0.6 \sim \mathrm{a}_{\mathrm{X}}=0.412 .
$$

6) A similar approach in the t-channel has been developed and for heavy particles and resonances. In all cases, in particular for a deuteron and helium-3, a deviation of a parameter " $a$ " as the masses ratio from $\mathrm{a}_{\mathrm{x}}=$ 0.412 accompanied by the suppression of contribution of the triangular anomalous Landau diagrams and as a consequence to a regarding smaller hypernucleus and supernucleus electroproduction.

7) In the field of high-energy was studied formation of Galactic spin shock-waves. In all cases above $20^{\circ}$ Galactic longitude intensity of diffuse gamma radiation quickly subsides, so that the theory of Eight spin model profile compared with the intensity of Galactic diffuse gamma radiation from large gamma-ray space telescope (GLAST) is an experimental distribution of diffuse Galactic Gamma-ray bursts very accurately, in all range of the Galactic longitude within the accuracy of measurements in the presence of extra-galactic sources, Sails, etc. All of this confirms the conservation low of the spin angular momentum, as part of a full and the isotropy of space-time across the Galaxy.

8) There is an impression that time variation of solar neutrino flux and all solar cosmic rays is not isotropic. Their peak value detected ground-based observatories as orbital motion around the Sun at the moment of crossing the Galactic directions of anisotropy in the projection on to the plane of the ecliptic. You can verify this by measurements of the solar constant and solar cosmic rays space stations operating in the orbit of Mercury, as well as on Mars. Peak values of existing data on Mercury do not have differences with this.

\section{References}

[1] A. G. Syromyatnikov. Comparison Analogy between Properties of Hypernucleus and Supernucleus with Properties of the Elementary Particles and Resonances Electroproduction by Spin Shock Waves. International Journal of High Energy Physics. Special Issue: Breaking of Space Symmetry in the Masses Spectrum Problem. Volume 3, Issue 1, January 2016, Published: Mar. 14, 2016 Pages: 1-8. DOI: 10.11648/j. ijhep.s.2016030101.12.

[2] A. G. Syromyatnikov. When Anisotropy of Vacuum Set The Space Isotropy. International Journal of High Energy Physics. Special Issue: Breaking of Space Symmetry in the Masses Spectrum Problem. Volume 3, Issue 1, January 2016, Published: Mar. 14, 2016 Pages: 9-14. DOI: 10.11648/j. ijhep.s.2016030101.12.

[3] A. G. Syromyatnikov. LXV International Conference "Nucleus2015". New horizons in nuclear physics, nuclear engineering, femto-and nano technologies. Book of abstracts. June 29 - July 3, 2015, Saint-Petersburg, Russia. P. 173.

[4] A. G. Syromyatnikov On the Problem of Neutrino Mass in the Aspect of the Detected Spatial Anisotropy of Temporal Variations of Solar Neutrinos Flux, American Journal of Aerospace Engineering, Published Online: Oct. 15, 2015 Pages: 11-29. DOI: 10.11648/j.ajae.20150203.11

[5] A. G. Syromyatnikov. Physical effects in Conformal Gauge Theory of Gravitation (LAP Lambert Academic Publishing GmbH \& Co. KG, Saarbrucken, Germany, 2012).

[6] A. G. Syromyatnikov. On Similarity between All-Known Elementary Particles and Resonances Mass Spectrum and Nuclear Atomic Weight. Univ. J. Phys. And Appl. 2(2) 76-79. DOI: 10.13189/ujpa.2014.02023.

[7] A. G. Syromyatnikov. Interaction in quantum gravitating systems, Vestnik Sanct Peterburgskogo universiteta. Ser. 4.Vip. 4 (2009) 410-425.

[8] A. G. Syromyatnikov. On some feature of possible torsion effects on observables at hadron colliders, Int. J. Geom. Methods Mod. Phys Vol. 12, No. 10 (2015) 1550080 (pages 147) DOI: $10.1142 / \mathrm{S} 0219887815500802$. 